\title{
Selective Determination of Acidic Drugs in Water Samples Using Online Solid Phase Extraction Liquid Chromatography with Alginate Incorporated Multi-Walled Carbon Nanotubes as Extraction Sorbent
}

\author{
Nurzaimah Zaini @ Othman ${ }^{1}$, Nor Suhaila Mohamad Hanapi ${ }^{1, *}$, Nor'ashikin Saim ${ }^{1}$, \\ Wan Nazihah Wan Ibrahim ${ }^{1}$, and Ahmad Lutfi Anis ${ }^{2}$ \\ ${ }^{1}$ Faculty of Applied Sciences, Universiti Teknologi MARA, 40450 Shah Alam, Selangor, Malaysia \\ ${ }^{2}$ Faculty of Applied Sciences, Universiti Teknologi MARA, 94300 Kota Samarahan, Sarawak, Malaysia
}

*Corresponding author:

tel: $+603-55435586$

email:norsuhaila979@salam.uitm.edu.my

Received: February 20, 2019

Accepted: July 26, 2019

DOI: $10.22146 /$ ijc. 43703

\begin{abstract}
A rapid and effective method is developed for selective determination of five selected acidic drugs (salicylic acid, naproxen, diclofenac, ibuprofen and mefenamic acid) in water samples by using online solid phase extraction (Online-SPE) prior to liquid chromatography diode array detector (LC-DAD) analysis. In this study, Alginate incorporated multi-walled carbon nanotubes (Alg-MWCNT) beads were prepared and utilized as solid phase extraction sorbent. Optimization of online SPELC operating parameters such as valve switching time, composition of acetonitrile and buffer $\mathrm{pH}$ was conducted using Box-Behnken Design of Response Surface Methodology (RSM) to evaluate the interactive effects of these three variables. Under the optimized conditions (valve switching time: $1.5 \mathrm{~min}$, composition of acetonitrile: MSA, 60:40 and buffer $p H$ : $p H 2)$, the method showed good linearity $\left(1-500 \mu \mathrm{g} \mathrm{L}^{-1}\right)$ with coefficient of determination $\left(R^{2}\right)$ of 0.9971-0.9996 and low limits of detection $\leq 0.018 \mu \mathrm{g} \mathrm{L} \mathrm{L}^{-1}$. The method showed high relative recoveries in the range of 75-110\% for river water and tap water samples, respectively with RSDs of $\leq 7.8(n=3)$. This method was successfully applied to the determination of acidic drugs in river and tap water samples. In addition, Alg-MWCNT sorbent offered high degree of selectivity and efficiency for online SPE-LC-DAD analysis.
\end{abstract}

Keywords: online SPE-LC; alginate; MWCNTs; acidic drugs; water samples

\section{- INTRODUCTION}

One of the trends in drug analysis nowadays is the development of rapid, highly efficient, as well as sensitive and reliable method for the identification and quantification of analytes in various matrices. Recent developments and improvements in analytical methodologies and advanced instruments have made the detection of pharmaceutical drugs at low concentration level possible. As a result of this advancement, pharmaceutical residues can be detected in various environmental samples and recognized as an 'emerging' contaminant [1].

In the last few decades, pharmaceutical drugs have played an increasingly important role in improving the quality of human life. Thousand tonnes of pharmaceuticals are consumed each year to treat and prevent illnesses [2]. However, the increasing consumption may lead to an accumulation of unused medicines in the household area. Nearly one-third of the pharmaceuticals sold annually are unused [3]. This situation may put human and animal health at risk due to accidental ingestion or exploitation of unused medicine.

Non-steroidal anti-inflammatory drugs (NSAIDs) can be present in water supplies, either from domestic or industrial waste water discharges. The common method used for analyzing these pharmaceuticals in trace level is chromatography, either gas chromatography or liquid chromatography coupled with sensitive detection technique which is mass spectrometry [4-8]. However, 
the matrix effects and the selection of internal standards should be taken into consideration [9]. Even though the concentrations of NSAIDs detected in a surface or ground water are in a range of $\mathrm{n} \mathrm{L}^{-1}$, they persist for longer periods of time in soils and sediments [10]. Therefore, the challenge remains in reaching these levels of detection using a method that is affordable, simple and yields reliable results.

In this study, a method of using online solid phase extraction liquid chromatography (online SPE-LC) for the separation of five selected pharmaceutical drugs was proposed. Quantitative, non-destructive and time saving are some criteria of an ideal extraction system [11]. Solid phase extraction (SPE) is the most common method used for the extraction of pharmaceutical drugs in water samples. SPE has been claimed as an effective sample preparation method for removal of interfering compounds and enrichment of analytes [12]. Over time, the SPE technique not only can be performed in the offline mode but also provides the possibility of online coupling to other analytical steps, such as chromatographic analysis. The application of online coupling with chromatographic analysis may shorten the analysis time, reduce sample contamination and analyte loss, as well as improve the precision and accuracy [13].

Carbon nanotubes (CNTs) possess advantages in adsorbent-adsorbate interactions. On the other hand, this potential sorbent has high production cost, involves nonbiodegradable materials and leads to some environmental issues. Meanwhile, alginate is a potential bio-sorbent for the removal of pharmaceutical drugs from water sample. However, it is readily soluble in aqueous media due to its hydrophilic nature which limits its application as an adsorbent.

Hence, this research pursues the potential usage of MWCNTs incorporated with alginate as a promising material to overcome both weaknesses with the aid of online-SPE-LC which is a simpler, more rapid and efficient method compared to the traditional offline SPE.

\section{- EXPERIMENTAL SECTION}

\section{Materials}

Ibuprofen, naproxen, diclofenac, mefenamic acid and salicylic acid were purchased from Sigma-Aldrich (purity assay in range of 98-101\%). Acetonitrile (ACN) and methanol $(\mathrm{MeOH})$ of high performance liquid chromatography (HPLC) grade was obtained from Merck (Darmstadt, Germany). Calcium chloride $\left(\mathrm{CaCl}_{2}\right)$ was obtained from $\mathrm{HmbG}$ Chemicals (Germany) and methanesulfonic acid (MSA) was procured from Sigma-Aldrich (St. Louis, USA). Ultrapure water was produced by Barnstead Nanopure (Thermo Scientific). Sodium hydroxide and hydrochloric acid were obtained from Merck, Darmstadt, Germany and sodium alginate from Qrec (New Zealand). A multi-walled carbon nanotubes (MWCNTs) with specific surface area $>233 \mathrm{~m}^{2} / \mathrm{g}$, purity of $>95 \%, 8-15 \mathrm{~nm}$ outer diameter $\times 50 \mu \mathrm{m}$ in length was purchased from Sun Nanotech (Jiangxi, China).

\section{Procedure}

\section{Preparation of standard and sample solutions}

The individual stock solution $\left(1000 \mathrm{mg} \mathrm{mL}^{-1}\right)$ of ibuprofen (IBU), naproxen (NAP), diclofenac (DIC), mefenamic acid (MEF) and salicylic acid (SAL) were prepared separately in methanol. All standard solutions were stored in the amber glass bottle at $4{ }^{\circ} \mathrm{C}$ when not in use. A series of working standard solutions were prepared in methanol by dilution prior to analysis to prevent from decomposition of analytes.

The water samples were collected in bottles, precleaned with acetone and filtered through a nylon membrane filter to remove colloidal particles and stored in a freezer at $4{ }^{\circ} \mathrm{C}$ until analysis commenced. The tap water and river water samples $(10 \mathrm{~mL}, \mathrm{pH} 3)$ were spiked with the standard solution of five pharmaceuticals NSAIDs mixture to give a final concentration of $0.1 \mu \mathrm{g} \mathrm{mL} \mathrm{L}^{-1}$ for each analyte.

\section{Preparation of Alg-MWCNT sorbent}

The preparation method for composite beads was adapted and modified from literature [14-15]. The composite beads were formed by suspension technique. Initially, 3\% $(\mathrm{w} / \mathrm{v})$ of sodium alginate solution was prepared under stirring at $60{ }^{\circ} \mathrm{C}$. Meanwhile, $0.3 \mathrm{~g}$ of MWCNT was dispersed in $30 \mathrm{~mL}$ deionized water under sonication for $30 \mathrm{~min}$. Then, MWCNT was added into the sodium alginate solution and sonicated for another 
$1 \mathrm{~h}$. The mixture solution was dripped through an injection needle into $1000 \mathrm{~mL}$ of $4 \%(\mathrm{w} / \mathrm{v})$ of calcium chloride solution. The alginate-MWCNT beads were formed upon contact with calcium ions. After removal from the calcium chloride bath, the beads were rinsed thoroughly with deionized water using $11 \mu \mathrm{m}$ filter paper and dried in an oven at $50{ }^{\circ} \mathrm{C}$ for $24 \mathrm{~h}$. The dried AlgMWCNT beads $(0.3 \mathrm{~g})$ were packed in an online SPE empty column, ready to be used for analysis.

The alginate-MWCNT beads were then characterized using Fourier Transformed Infrared (FTIR) spectroscopy (Thermo Fischer Scientific) in the range of $4000-400 \mathrm{~cm}^{-1}$ using Attenuated Total Reflection (ATR) technique with diamond as the ATR crystal. This method was used to determine the surface chemistry and components of the biopolymer composite beads. The functional groups were determined based on specific broad bands observed. Surface morphology of the AlgMWCNT sorbent was analyzed using an ultra-high resolution field emission electron microscope (FESEM) model Zeiss Supra 40VP with $10 \mathrm{~K}$ magnification. Nitrogen adsorption and desorption isotherm of the adsorbent were measured at $-196{ }^{\circ} \mathrm{C}$ using BELSORPmini II instrument (BEL. Japan Inc.). Prior to measurements, the samples were degassed at $70^{\circ} \mathrm{C}$ for $24 \mathrm{~h}$ in a vacuum oven. The isotherm was further analyzed using Brunauer-Emmett-Teller (BET) method to determine the specific surface area, total pore volume and average pore diameter of the sorbent.

\section{Online solid phase extraction system}

All analyses were performed using an automated high performance liquid chromatography Dionex Ultimate 3000 (Sunnyvale, CA, USA) system. The system comprises of a large volume loop (10.2 $\mathrm{mL}$ ) autosampler, dual gradient pump, left and right, a solvent rack with an integrated vacuum degasser, a thermostat column compartment, two columns (online SPE column and analytical column) and a diode array detector (DAD).

The analytical column was an Acclaim Polar Advantage II $(5 \mu \mathrm{m}, 120 \AA$ A $4.6 \times 150 \mathrm{~mm})$ (Thermo Scientific USA) and the online SPE clean-up was performed using alginate incorporated multi-walled carbon nanotube biopolymer sorbent packed in an empty online SPE column. The system was equipped with a programmable 6 port $/ 2$ position switching valve for several modes (loading, clean-up, elution and separation). Data were processed by the Chromeleon ${ }^{\mathrm{m}}$ Software v.6.8 (Dionex). Fig. 1 shows the schematics of the online SPE-LC system.

The method comprises three steps which are sample loading, clean-up, elution and LC separation. Both pumps ran simultaneously. The flow rate was set at $1 \mathrm{~mL} \mathrm{~min}{ }^{-1}$ throughout the analysis with a temperature of $40{ }^{\circ} \mathrm{C}$.

At equilibrium mode, $10 \mu \mathrm{L}$ of spiked water sample was loaded onto the flow system using auto-sampler (fitted with a $100 \mu \mathrm{L}$ syringe). SPE column was then positioned into the loading mode using the switching valve. The left pump was used to load sample from the

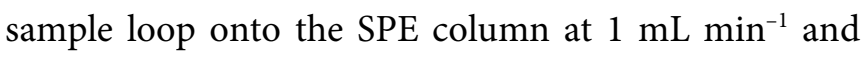
simultaneously the analytical column was equilibrated with the right pump. Next, the co-retained sample matrix was flushed out using a washing composition of $10 \mathrm{mM}$ MSA and ACN (95:5) while analytes were retained on the SPE column. During the cleanup process, the choice of mobile phases and time of switching valve are important factors that influence the extraction recovery [16]. After sample loading, the valve was switched to clean-up using the clean-up mobile phase (ACN: $10 \mathrm{mM}$ MSA, 5:95 (v/v) and kept for $1.0 \mathrm{~min}$ to remove any possible impurities retained together with analytes.

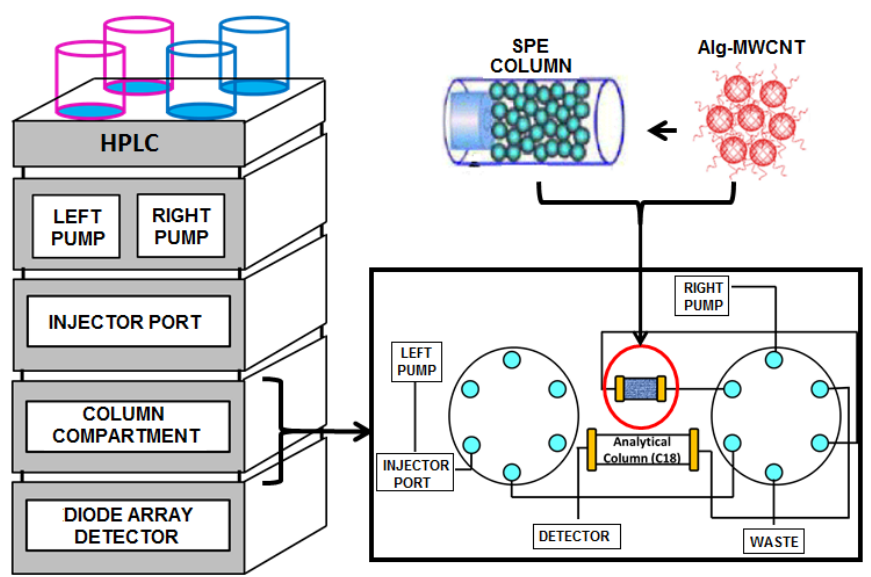

Fig 1. Schematic Diagram for Alg-MWCNT-OnlineSPE-LC 
In the elution step, the switching valve was switched into the elution position which couples the SPE column with the analytical column. The analytes were transferred using gradient elution mobile phase composition. Lastly, in the separation step the switching valve was switched back into equilibration mode and disconnected the SPE column from the analytical column. In this mode, analytes continued separating in the analytical column using the right pump. In the left pump, the SPE column was equilibrated prior to the next sample being loaded into the sample loop.

\section{Detection method}

All analytes were simultaneously analyzed using diode array detector (DAD) at various wavelengths. The DAD was set at $230 \mathrm{~nm}$. Identification of NSAIDs was based on retention time and ultraviolet (UV) spectrum of each analyte.

\section{- RESULTS AND DISCUSSION}

\section{Physical Properties of the Prepared Alg-MWCNT Beads}

Before the drying process, the shape of the composite beads formed were spherical with the turgidity coming from the formation of bivalent ions when alginate comes in contact with calcium ions [17]. Approximated size of the bead was $\sim 2 \mathrm{~mm}$ in diameter. After the drying process, the composite beads shrunk by half ( $\sim 1 \mathrm{~mm}$ in diameter) from the original size of the beads before drying as the water content in the beads had been removed through evaporation.

\section{Characterization of Alg-MWCNT Sorbent by FESEM Analysis}

Fig. 2(a-c) show the FESEM images of alginate, MWCNTs and Alg-MWCNT. Surface structure of alginate (Fig. 2(a)) under the magnification of $10 \mathrm{~K}$ shows a very smooth and clear surface. Meanwhile, the structure of MWCNT (Fig. 2(b)) under the same magnification shows some ball-of-string-like structure clumping together in random manner. It was also noticed that there were numerous entangled carbon nanotubes observed. The structure of the synthesized Alg-MWCNT (Fig. 2(c)) under the same magnification showed that the clumps of MWCNTs have been deposited onto the surface of the alginate. From the micrograph, it can be suggested that the MWCNTs particles had bound around the alginate surface [18].
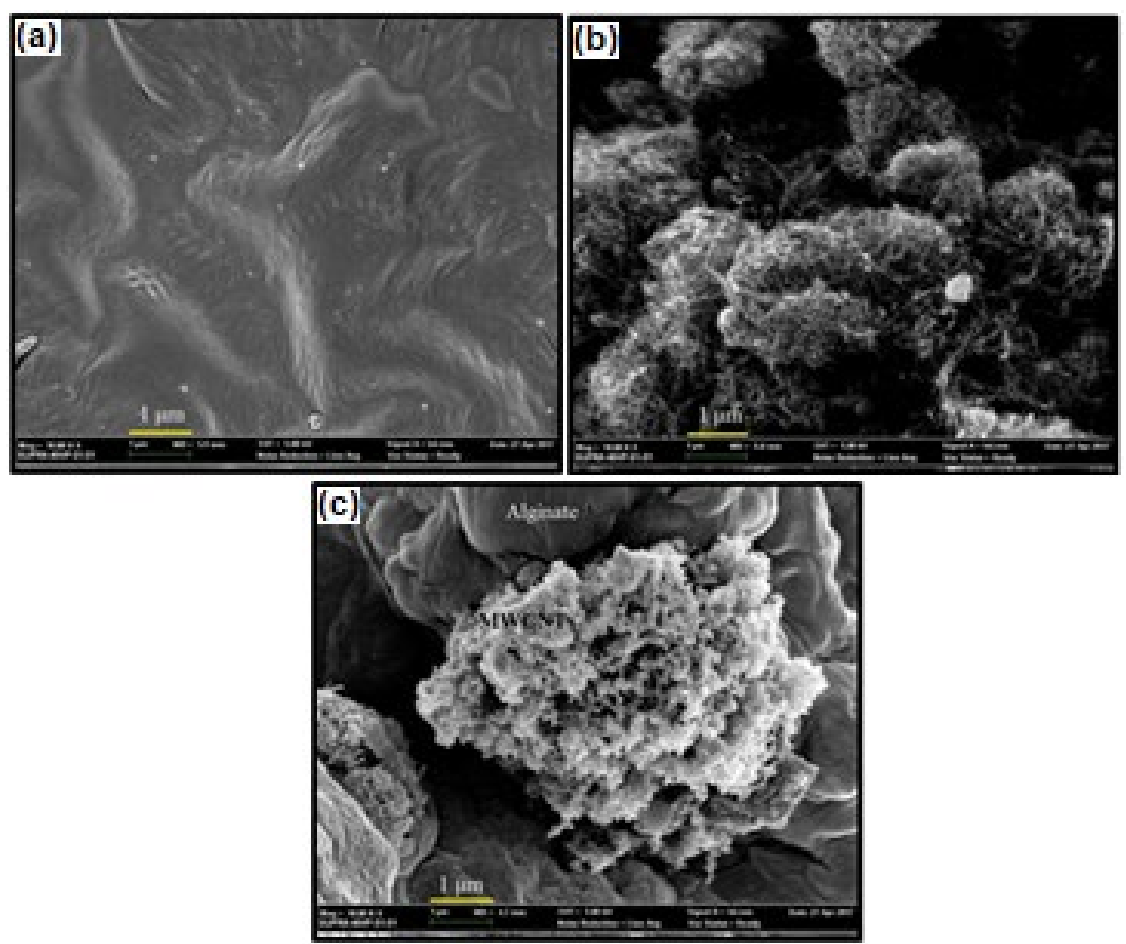

Fig 2. FESEM Micrographs of (a) Alginate (b) MWCNTs (c) Alg-MWCNT under 10 K Magnification 


\section{Characterization of Alg-MWCNT Sorbent by FTIR Analysis}

Fourier Transform Infrared (FTIR) spectroscopy was used to investigate functionalized spectra of sodium alginate, multi-walled carbon nanotubes (MWCNTs) and alginate-MWCNTs. The results for FTIR spectroscopy were recorded and compared. Fig. 3(a) shows the spectra for the three compounds.

Spectrum of sodium alginate showed important absorption bands corresponding to hydroxyl, ether, and carboxylic functional groups. Stretching vibrations of $\mathrm{O}-\mathrm{H}$ bonds of alginate appeared in a very broad band at $3258 \mathrm{~cm}^{-1}$. Observed bands in 1594 and $1407 \mathrm{~cm}^{-1}$ were attributed to the stretching vibrations of carbon-oxygen double bond and carbon-carbon double bond, respectively. Meanwhile, the band at $1026 \mathrm{~cm}^{-1}$ could be attributed to the $\mathrm{C}-\mathrm{O}$ stretching vibration.

On the other hand, the IR spectrum of carboxylated multi-walled carbon nanotubes (MWCNTs) displays some unresolved peaks due to the low transparency of CNT that caused it difficult for IR ray to penetrate and be absorbed into the sample. A broad peak appeared at $3305 \mathrm{~cm}^{-1}$ corresponding to the $\mathrm{O}-\mathrm{H}$ bond stretch of carboxylic acid functional group of MWCNTs. A peak appeared at $1772 \mathrm{~cm}^{-1}$ which represents $\mathrm{C}=\mathrm{O}$ from carboxylate group. Peaks at 1645 and $1405 \mathrm{~cm}^{-1}$ suggest that the compound has a benzene ring which is the main structure of MWCNTs.

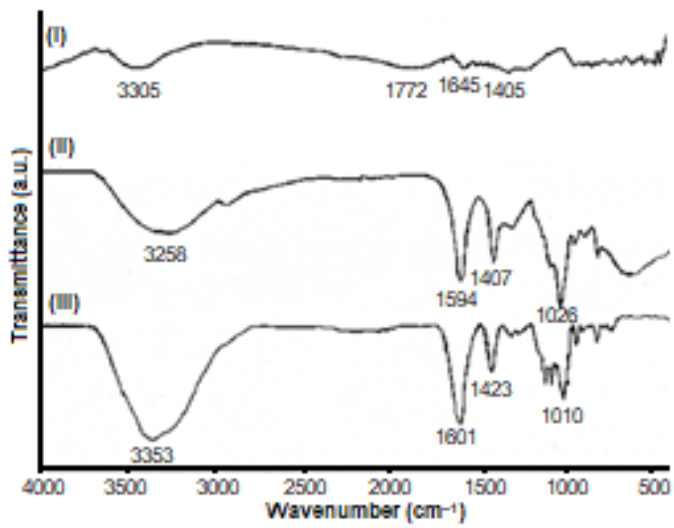

(a)

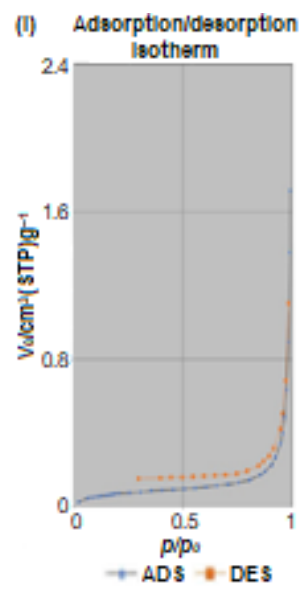

$A D S=-D E S$

The FTIR spectrum of alginate incorporated multiwalled carbon nanotube shows slightly different peaks as compared to the alginate spectrum. It is suggested that the hydroxyl group present at $3258 \mathrm{~cm}^{-1}$ in the alginate spectra had been shifted to $3353 \mathrm{~cm}^{-1}$ in the AlgMWCNT spectra and the peak is broader than in the alginate spectra, indicating that the carboxyl group of MWCNT and the carboxyl group from sodium alginate were cross-linked synchronously with calcium ions [19]. Apart from that, this also shows that the alginate was cross-linked by the aid of calcium chloride solution [20].

The adsorption bands for carbon-oxygen double bond and carbon-carbon double bond were shifted from 1594 and $1407 \mathrm{~cm}^{-1}$ to 1601 and $1423 \mathrm{~cm}^{-1}$, respectively. It was also observed that the carbon-oxygen single bond band at $1026 \mathrm{~cm}^{-1}$ in the alginate spectra was less intense in the Alg-MWCNT spectra, and the peak was shifted to $1010 \mathrm{~cm}^{-1}$. This suggests that there could be substitution reaction that may have occurred during the formation of Alg-MWCNT [21].

\section{Isotherm and Surface Area Analysis of Alg- MWCNT Sorbent}

Nitrogen adsorption and desorption isotherm of the adsorbent were measured at $-196{ }^{\circ} \mathrm{C}$ using BELSORP-mini II instrument (BEL. Japan Inc.). Prior to these measurements, the samples were degassed at $70{ }^{\circ} \mathrm{C}$ for $24 \mathrm{~h}$ in a vacuum oven. The isotherm was further analyzed using Brunauer-Emmett-Teller (BET) method

Fig 3. (a) Infrared spectra of (i) MWCNTs, (ii) Alginate and (iii) Alg-MWCNT and (b) Brunauer Emmet Teller (BET) analysis of (i) Alginate, (ii) MWCNTs and (iii) Alg-MWCNT bead 
to identify the specific surface area, total pore volume, and average pore diameter of the adsorbent. The porosity and surface area of pristine alginate, MWCNTs and AlgMWCNT beads were determined by BET. The BET analysis provides the value of specific surface area, total pore volume and average pore diameter while pore analysis provides information on the pore distribution of the adsorbents. Nitrogen adsorption-desorption isotherm of pristine alginate, MWCNTs and Alg-MWCNT (Fig. 3(b)) at $77 \mathrm{~K}$ shows that they possess Types IV hysteresis loop which indicates a mesoporous to macroporous adsorbent with strong affinities towards adsorbate [22].

Table 1 shows the specific surface area, total pore volume and average pore diameter obtained from BET analysis. The results show that the pristine alginate has lower surface value than MWCNTs. The results show that the incorporation of MWCNTs in alginate matrix increases the surface area, and total pore volume of the Alg-MWCNT beads. The low surface area for AlgMWCNT is probably due to the low amount of MWCNTs used in the composition of beads (3\%).

\section{Sorbent-Sorbate Interactions between Alg-MWCNT with Target Analytes}

The possible interaction between the sorbents (alginate and MWCNTs) and the interaction between the sorbent with the five target analytes were shown in Fig. 4. Strong hydrogen bonding interactions can occur between side chains of biopolymers alginate with other molecules either with the multi-walled carbon nanotubes or with the analytes. The highly associated interaction between blended Alg-MWCNT through hydrogen bonding helped improve swelling and gelation properties of alginate while imparting mechanical strength to agar. Meanwhile, the hydrogen bonding between alginate and target analytes can contribute to the extraction efficiency. On the other hand, $\pi-\pi$ interaction may occur between the MWCNTs and the target analytes. At lower $\mathrm{pH}$, strong interactions between the non-polar aromatic bonds of targeted analytes towards the hexagonal array of graphene sheets of MWCNTs could be attributed to $\pi-\pi$ and hydrophobic interactions [23-25].

\section{Optimization of Online SPE-LC Parameters Using Box-Behnken Design (BBD)}

Optimization for the extraction of five types of NSAIDs using online solid phase extraction yielded three optimized conditions, which are $1.5 \mathrm{~min}$ for valve switching time, $60 \%$ composition of acetonitrile in elution solvent composed of ACN: MSA and $\mathrm{pH} 2$ for buffer with desirability 0.974 . The regression equation of the fitted model is as shown in Eq. (1), where $Y$ is the response (total peak area) of target analytes, $\mathrm{A}$ is the valve switching time, $\mathrm{B}$ is acetonitrile composition and $\mathrm{C}$ is buffer $\mathrm{pH}$.

$$
\begin{aligned}
\mathrm{Y}= & 9.62-0.037 \mathrm{~A}-0.009 \mathrm{~B}+0.30 \mathrm{C}-0.31 \mathrm{~A}^{2}-0.30 \mathrm{~B}^{2} \\
& -0.35 \mathrm{C}^{2}-0.062 \mathrm{AB}+0.081 \mathrm{AC}-0.060 \mathrm{BC}
\end{aligned}
$$

The statistical significance of the terms used in the model was defined via ANOVA analysis. A multi-linear regression analysis was applied to the results of BoxBehnken design. Composition of mobile phase and valve

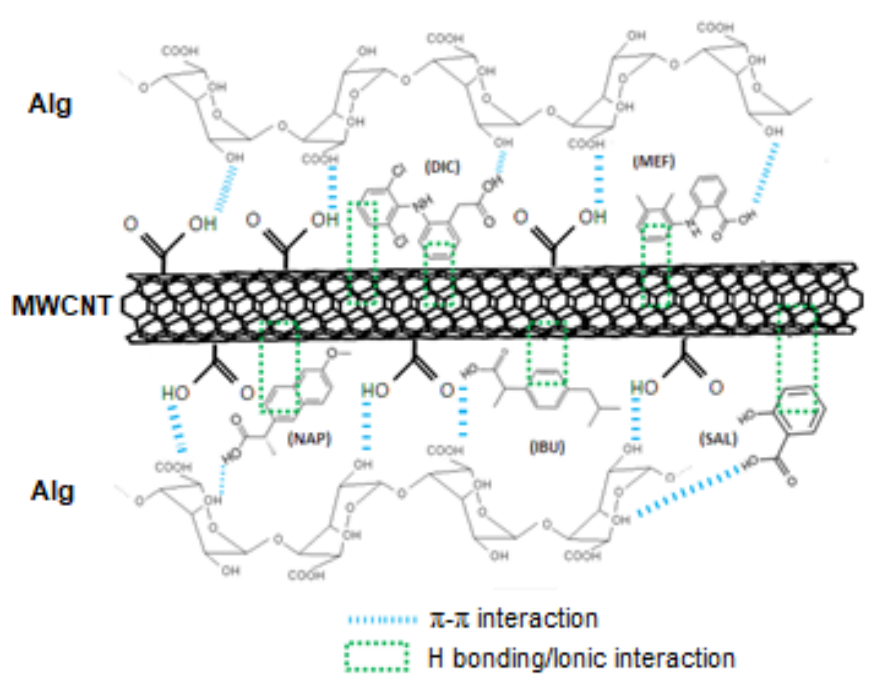

Fig 4. Sorbent-sorbate interactions between AlgMWCNT with target analytes

Table 1. Results for BET analysis

\begin{tabular}{lccc}
\hline Sample & Pristine alginate & MWCNTs & Alg-MWCNT \\
\hline sBET $\left(\mathrm{m}^{2} \mathrm{~g}^{-1}\right)$ & 0.28363 & 134.14 & 0.62756 \\
Total pore volume $\left(\mathrm{cm}^{3} \mathrm{~g}^{-1}\right)$ & 0.0024974 & 1.4358 & 0.0048269 \\
Average pore diameter $(\mathrm{nm})$ & 35.221 & 42.817 & 30.766 \\
\hline
\end{tabular}


switching time are two significant variables for the cleanup step [26]. The effect of independent variables, which are valve switching time, acetonitrile composition, and buffer $\mathrm{pH}$ were evaluated by second order (quadratic). Table 2 shows the analysis of variance (ANOVA) regression model for response quadratic model for salicylic acid, naproxen, ibuprofen, diclofenac and mefenamic acid. The reliability of the fitted model was proven by the high F-values and the low $\mathrm{P}$-values.

The quality of fit on the quadratic polynomial model was presented by the coefficient of determination, $\mathrm{R}^{2}$ [27]. The value of $\mathrm{R}^{2}$ shows that there is an acceptable relationship between the predicted and actual values (Fig. 5(a)). The closer the value of $\mathrm{R}^{2}$ to unity, the better is the empirical model fit to the actual data. Criteria for a good fit of a model, the $\mathrm{R}^{2}$ should be at least 0.80 [28]. The $\mathrm{R}^{2}$ value calculated for the extraction of five NSAIDs was 0.9052, indicating a significantly good fitted model.

The main effects of the variables were visualized by Pareto chart (Fig. 5(b)). According to this figure, the acetonitrile composition affects the extraction the most. This is because, among the three parameters being investigated, acetonitrile composition is very crucial to ensure that the analyte will be well-eluted. The most commonly used organic solvents in HPLC are acetonitrile as it results in the lowest system backpressure in water mixtures and has a very low UV cut off for better UV/Vis detection sensitivity [29].

\section{Effect of valve switching time on the peak of analytes}

The right time to switch the valve is very important to ensure all the analytes have been fully extracted by the sorbent and ready for separation step. Effect of valve switching time depends on the step that has been done in the SPE column. For example, if the valve has been switched too early, the analyte would not have been fully extracted by the SPE column. Some analytes would have skipped the SPE column and directly flowed into the analytical column during the separation step. The result will either be low in recoveries or some analytes may not be eluted (Fig. 6(a)).

\section{Effect of ACN:MSA composition on the peak of analytes}

In optimizing solvent composition, various isocratic elution of acetonitrile and $10 \mathrm{mM}$ MSA were studied. Each analyte were eluted by increasing the strength of elution solvent. The increase in acetonitrile composition means that the elution strength of the

Table 2. Summary of ANOVA analysis of NSAIDs

\begin{tabular}{lllccl}
\hline Transform & Model & Lack of fit & DF & R-square & \multicolumn{1}{c}{ Equation } \\
\hline Square Root & Quadratic & Not significant & 9 & 0.9052 & Sqrt (Total Peak Area) $=$ \\
& Significant & & & & $9.62-0.037 \mathrm{~A}-0.009 \mathrm{~B}+0.30 \mathrm{C}-0.31 \mathrm{~A}^{2}-0.30 \mathrm{~B}^{2}$ \\
& & & & $-0.35 \mathrm{C}^{2}-0.062 \mathrm{AB}+0.081 \mathrm{AC}-0.060 \mathrm{BC}$ \\
\hline
\end{tabular}

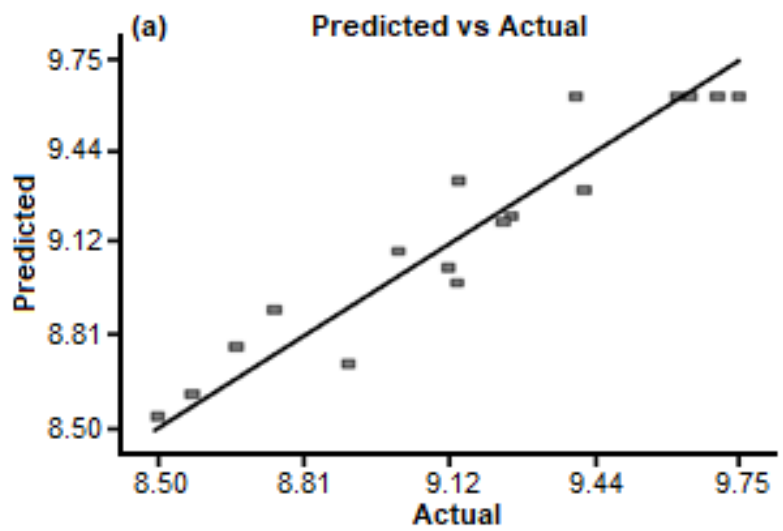

(b)

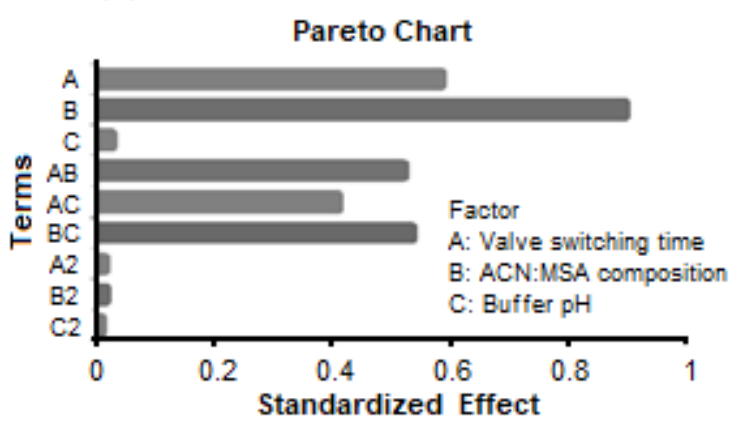

Fig 5. (a) The parity plot between predicted and actual (experimental) values for all analytes, (b) Pareto chart of the standardized effects in Alg-MWCNT online SPE-LC 

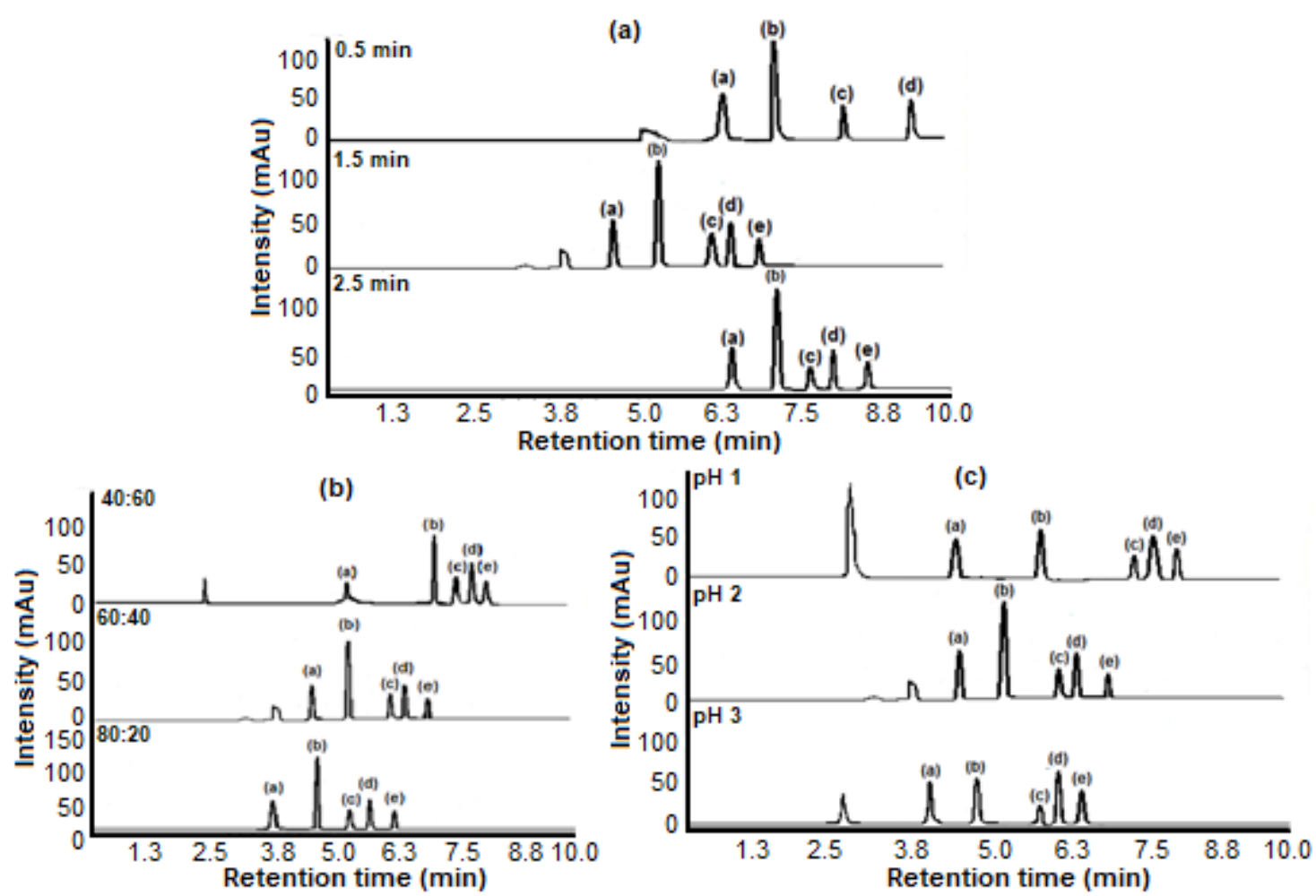

Fig 6. Effect of (a) Valve switching time, (b) ACN:MSA composition and (c) Buffer pH on the peak of analytes, (a) salicylic acid, (b) naproxen, (c) diclofenac, (d) ibuprofen and (e) mefenamic acid

retained analyte on the sorbent will be increased. All of the analytes were eluted from the column within 60-70\% of ACN. A decrease in composition of $10 \mathrm{mM}$ MSA with increasing ACN composition, improved the separation of the NSAIDs. Fig. 6(b) shows that the increase in the strength of the elution solvent (ACN:MSA) is proportional to the total peak area of the analytes.

\section{Effect of buffer $\mathrm{pH}$ on the peak of analytes}

Buffering is commonly needed when analyzing ionizable analytes with reversed phase LC. The ionized species always elute from the column earlier. Sometimes buffer is needed because some impurities are ionizable. The most suitable buffer $\mathrm{pH}$ for acidic analyte is at least two units below the pKa of the analyte. As shown in Fig. 6(c), at $\mathrm{pH} 2$, the peak area of analytes were higher compared to $\mathrm{pH} 1$. However, the peak area of analytes at $\mathrm{pH} 3$ decreased as there could be some analytes that were still in ionized form and combined with the impurities [30].

\section{Response Contour Plot}

Fig. 7(a) shows the three-dimensional contour plot for the valve switching time and the buffer $\mathrm{pH}$ against the total peak area of the analytes. Meanwhile, the third parameter, which is composition of acetonitrile were kept at a mid-constant level: $60 \%$. As observed, the hyperbolic contour plot shows that both buffer $\mathrm{pH}$ and valve switching time were correlated to one another. The optimal extraction efficiencies were observed near the middle of the contour plot at medium buffer $\mathrm{pH}$ and valve switching time which was located within the experimental region.

Fig. 7(b) shows the three-dimensional graph plot for the composition of acetonitrile and the buffer $\mathrm{pH}$ against the total peak area of the analytes. The third parameter which was valve switching time was kept at a mid-constant level: $1.5 \mathrm{~min}$. At lower $\mathrm{pH}$, strong interaction between the non-polar aromatic bonds of the targeted analytes toward the hexagonal arrays of graphene sheets of MWCNT could be attributed to $\pi-\pi$ and van hydrophobic interactions [31].

A three-dimensional graph was plotted for the valve switching time and acetonitrile composition against 

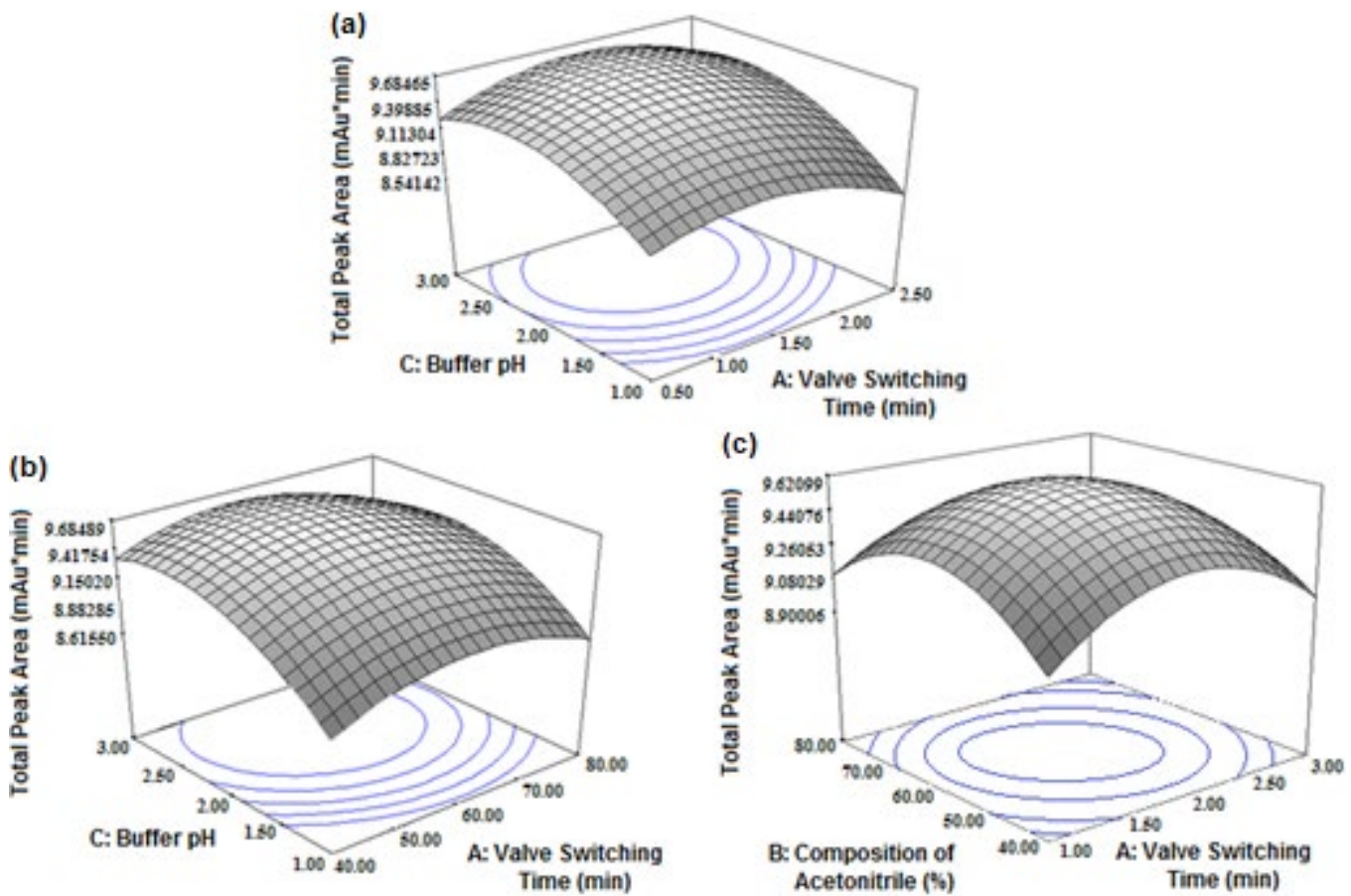

Fig 7. RSM 3-D contour plots for Alg-MWCNT-online SPE-LC. Legends: (a) pH buffer and valve switching time (b) $\mathrm{pH}$ buffer and $\mathrm{ACN}$ composition (c) $\mathrm{ACN}$ composition and valve switching time against peak area of analytes

the total peak area of the analytes, as shown in Fig. 7(c). The third parameter which was $\mathrm{pH}$ buffer was kept at a mid-constant level that is $\mathrm{pH}$ 2.0. It suggests that the optimum valve switching time is at $1.5 \mathrm{~min}$ and optimum acetonitrile composition is at $60 \%$. With increase in the strength of the elution solvent, there is an increase in the total peak area of analytes. At one level, the high acetonitrile composition and the total peak area was maintained and gradually started to decrease. It could be due to the $60 \%$ acetonitrile composition which provides the most suitable polarity strength for the analyte to be eluted.

\section{Method Validation and Analytical Performance of Online SPE-LC}

The optimization of online SPE-LC method was then validated for relative recoveries, sample calibration, and Limit of Detection (LOD). A calibration curve was generated using five concentrations of standard mixture in the range of 1 to $500 \mu \mathrm{g} \mathrm{L} \mathrm{L}^{-1}$ with three replicates. Linear curves for each analyte were obtained with a good

Table 3. Validation data of Alg-MWCNT-online SPE-LC method of NSAIDs in tap and river water samples

\begin{tabular}{clcccc}
\hline Sample & Analytes & $\begin{array}{c}\text { Linear range } \\
\left(\mu \mathrm{g} \mathrm{L}^{-1}\right)\end{array}$ & $\begin{array}{c}\text { Coefficient of } \\
\text { determination, } \mathrm{R}^{2}\end{array}$ & LOD $\left(\mu \mathrm{g} \mathrm{L}^{-1}\right)$ & $\begin{array}{c}\text { Precision (RSD, \%) } \\
(\mathrm{n}=3)\end{array}$ \\
\hline Tap water & Salicylic acid & $1-500$ & 0.9993 & 0.0183 & 4.9 \\
& Naproxen & $1-500$ & 0.9975 & 0.0118 & 5.0 \\
& Diclofenac & $1-500$ & 0.9991 & 0.0062 & 1.4 \\
& Ibuprofen & $1-500$ & 0.9996 & 0.0075 & 3.4 \\
& Mefenamic acid & $1-500$ & 0.9971 & 0.0141 & 3.8 \\
\hline River water & Salicylic acid & $1-500$ & 0.9984 & 0.0152 & 0.9 \\
& Naproxen & $1-500$ & 0.9992 & 0.0159 & 6.8 \\
& Diclofenac & $1-500$ & 0.9978 & 0.0176 & 1.7 \\
& Ibuprofen & $1-500$ & 0.9995 & 0.0144 & 2.5 \\
& Mefenamic acid & $1-500$ & 0.9983 & 0.0095 & 2.1 \\
\hline
\end{tabular}


correlation coefficient $\left(\mathrm{R}^{2}=0.9971-0.9996\right)$. The sensitivity of the method expressed as Limit of Detection (LOD) was calculated at a signal to noise ratio of 3 based on linear regression method and the results were in the range of $0.0062-0.0183 \mu \mathrm{g} \mathrm{L}{ }^{-1}$ for LODs. This proved that LOD for online SPE-LC method is much lower than traditional SPE method [32]. Table 3 shows the validation data for online SPE-LC of NSAIDs from water sample.

\section{Application of Online SPE-LC on River Water and Tap Water Samples}

To investigate the practicality of the proposed method for the analysis of acidic drugs in real samples, the method was applied to analyze salicylic acid, ibuprofen, naproxen, diclofenac and mefenamic acid in the tap and river water samples (Fig. 8). However, there was no peak detected for naproxen, ibuprofen, diclofenac and mefenamic acid in the samples. Percentage recovery study
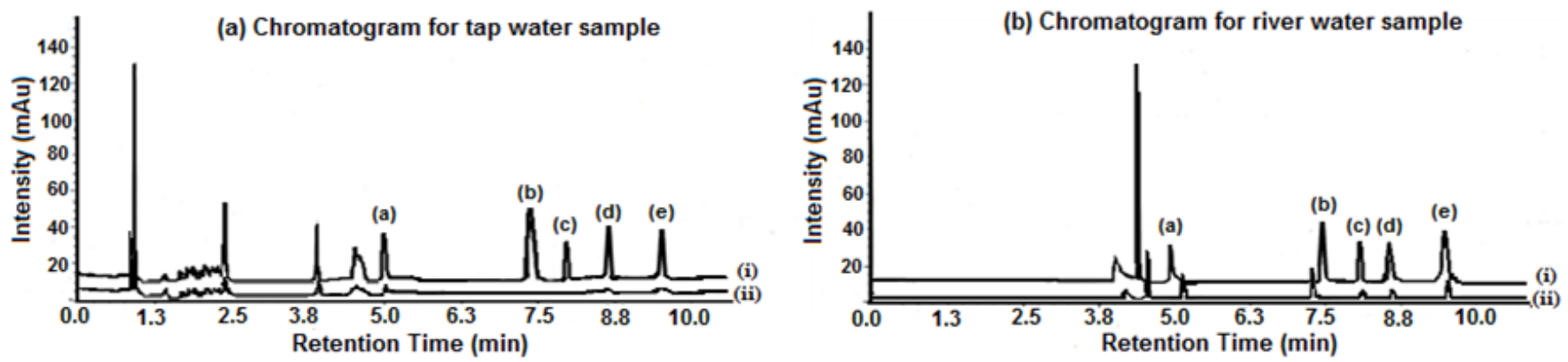

Fig 8. Chromatogram of online-SPE-LC of five NSAIDs in tap and river water samples; (i) spiked sample (ii) blank sample; (a) salicylic acid, (b) naproxen, (c) diclofenac, (d) ibuprofen and (e) mefenamic acid

Table 4. Relative recoveries (\%) and method precisions (RSD \%, $\mathrm{n}=3$ ) at two different concentrations of AlgMWCNT-online SPE-LC in tap water and river water samples

\begin{tabular}{llcc}
\hline \multirow{3}{*}{ Sample } & \multirow{2}{c}{ Analyte } & \multicolumn{2}{c}{ Average relative recovery, \% (RSD, \%) } \\
\cline { 3 - 4 } & & $50 \mu \mathrm{g} \mathrm{L}^{-1}$ & $100 \mu \mathrm{g} \mathrm{L}^{-1}$ \\
\cline { 2 - 4 } Tap water & Salicylic acid & $75(4.5)$ & $79(4.6)$ \\
& Naproxen & $91(4.1)$ & $96(7.8)$ \\
& Diclofenac & $76(3.7)$ & $78(5.5)$ \\
& Ibuprofen & $79(4.1)$ & $76(3.8)$ \\
& Mefenamic acid & $85(3.2)$ & $82(5.3)$ \\
\hline River water & Salicylic acid & $91(3.2)$ & $104(4.8)$ \\
& Naproxen & $83(3.9)$ & $86(2.7)$ \\
& Diclofenac & $104(4.3)$ & $110(0.9)$ \\
& Ibuprofen & $79(5.3)$ & $83(2.0)$ \\
& Mefenamic acid & $87(3.6)$ & $103(4.7)$ \\
\hline
\end{tabular}

Nurzaimah Zaini @ Othman et al. 
solid phase extraction combined with liquid chromatography (SPE-LC) has shown to have improved the extraction efficiency as it shortens the extraction and analysis time. Several parameters were optimized in the online SPE method. The optimum conditions were as follows: buffer $\mathrm{pH}$ at $\mathrm{pH} 2, \mathrm{ACN}$ : MSA composition at 60:40 and suitable valve switching time at $1.5 \mathrm{~min}$. The optimum parameters were used in the analysis of real samples. All five analytes were successfully extracted using the same conditions in online SPE-LC. Good linearities were achieved for the analytes with coefficients of determination, $\mathrm{R}^{2}$, in the range of $0.9971-0.9996$. The method was successfully applied for the analysis of river water and tap water samples, with good relative recoveries in the range of $75-110 \%$. Online SPE-LC method by using Alg-MWCNT as sorbent proved to be a rapid, selective and efficient technique for the extraction and separation of acidic drugs in aqueous matrices.

\section{- ACKNOWLEDGMENTS}

The authors would like to thank Universiti Teknologi MARA (UiTM), Shah Alam, Selangor for providing research facilities and the Ministry of Education Malaysia for financial support through the FRGS research grant 600-IRMI/FRGS 5/3 (010/2019).

\section{- REFERENCES}

[1] Białk-Bielińska, A., Kumirska, J., Borecka, M., Caban, M., Paszkiewicz, M., Pazdro, K., and Stepnowski, P., 2016, Selected analytical challenges in the determination of pharmaceuticals in drinking/marine waters and soil/sediment samples, $J$. Pharm. Biomed. Anal., 121, 271-296.

[2] Ji, Y., Du, Z., Zhang, H., and Zhang, Y., 2014, Rapid analysis of non-steroidal anti-inflammatory drugs in tap water and drinks by ionic liquid dispersive liquid-liquid microextraction coupled to ultra-high performance supercritical fluid chromatography, Anal. Methods, 6 (18), 7294-7304.

[3] Hanapi, N.S.M., Sanagi, M.M., Ibrahim, W.A.W., Saim, N., Ismail, A.K., Ibrahim, W.N.W., and Tahiruddin, S., 2015, Analysis of some antidepressant drugs in aqueous samples using agarose film micro-electro driven membrane extraction, Der Pharma Chem., 7 (5), 235-242.

[4] Kosjek, T., Heath, E., and Krbavčič, A., 2005, Determination of non-steroidal anti-inflammatory drug (NSAIDs) residues in water samples, Environ. Int., 31 (5), 679-685.

[5] Payán, M.R., López, M.Á.B., Torres, R.F., Navarro, M.V., and Mochón, M.C., 2011, Electromembrane extraction (EME) and HPLC determination of nonsteroidal anti-inflammatory drugs (NSAIDs) in wastewater samples, Talanta, 85 (1), 394-399.

[6] Zhang, Y., and Lee, H.K., 2012, Ionic liquid-based ultrasound-assisted dispersive liquid-liquid microextraction followed high-performance liquid chromatography for the determination of ultraviolet filters in environmental water samples, Anal. Chim. Acta, 750, 120-126.

[7] Yao, C., Li, T., Twu, P., Pitner, W.R., and Anderson, J.L., 2011, Selective extraction of emerging contaminants from water samples by dispersive liquid-liquid microextraction using functionalized ionic liquids, J. Chromatogr. A, 1218 (12), 1556-1566.

[8] Iuliani, P., Carlucci, G., and Marrone, A., 2010, Investigation of the HPLC response of NSAIDs by fractional experimental design and multivariate regression analysis. Response optimization and new retention parameters, J. Pharm. Biomed. Anal., 51 (1), 46-55.

[9] Hernando, M.D., Mezcua, M., Fernández-Alba, A.R., and Barceló, D., 2006, Environmental risk assessment of pharmaceutical residues in wastewater effluents, surface waters and sediments, Talanta, 69 (2), 334-342.

[10] Toledo-Neira, C., and Álvarez-Lueje, A., 2015, Ionic liquids for improving the extraction of NSAIDs in water samples using dispersive liquidliquid microextraction by high performance liquid chromatography-diode array-fluorescence detection, Talanta, 134, 619-626.

[11] Zhang, S.Q., Bi, H.M., and Liu, C.J., 2007, Extraction of bio-active components from Rhodiola sachalinensis under ultrahigh hydrostatic pressure, Sep. Purif. Technol., 57 (2), 277-282. 
[12] Hennion, M.C., 1999, Solid-phase extraction: method development, sorbents, and coupling with liquid chromatography, J. Chromatogr. A, 856 (1-2), 3-54.

[13] Chen, L., Wang, H., Zeng, Q., Xu, Y., Sun, L., Xu, H., and Ding, L., 2009, On-line coupling of solid-phase extraction to liquid chromatography-A review, $J$. Chromatogr. Sci., 47 (8), 614-623.

[14] Jeon, O., Powell, C., Ahmed, S.M., and Alsberg, E., 2010, Biodegradable, photocrosslinked alginate hydrogels with independently tailorable physical properties and cell adhesivity, Tissue Eng. Part A, 16 (9), 2915-2925.

[15] Sahasathian, T., Praphairaksit, N., and Muangsin, N., 2010, Mucoadhesive and floating chitosan-coated alginate beads for the controlled gastric release of amoxicillin, Arch. Pharmacal Res., 33 (6), 889-899.

[16] Fernández-Ramos, C., Šatínský, D., Šmídová, B., and Solich, P., 2014, Analysis of trace organic compounds in environmental, food and biological matrices using large-volume sample injection in column-switching liquid chromatography, TrAC, Trends Anal. Chem., 62, 69-85.

[17] Sakai, S., and Kawakami, K., 2007, Synthesis and characterization of both ionically and enzymatically cross-linkable alginate, Acta Biomater, 3 (4), 495-501.

[18] Joddar, B., Garcia, E., Casas, A., and Stewart, C.M., 2016, Development of functionalized multi-walled carbon-nanotube-based alginate hydrogels for enabling biomimetic technologies, Sci. Rep., 6, 32456.

[19] Jie, G., Kongyin, Z., Xinxin, Z., Zhijiang, C., Min, C., Tian, C., and Junfu, W, 2015, Preparation and characterization of carboxyl multi-walled carbon nanotubes/calcium alginate composite hydrogel nano-filtration membrane, Mater. Lett., 157, 112-115.

[20] Pawar, S.N., and Edgar, K.J., 2012, Alginate derivatization: A review of chemistry, properties and applications, Biomaterials, 33 (11), 3279-3305.

[21] Şolpan, D., and Torun, M., 2005, Investigation of complex formation between (sodium alginate/ acrylamide) semi-interpenetrating polymer networks and lead, cadmium, nickel ions, Colloids Surf., A, 268 (1-3), 12-18.
[22] Sangwichien, C., Aranovich, G.L., and Donohue, M.D., 2002, Density functional theory predictions of adsorption isotherms with hysteresis loops, Colloids Surf., A, 206 (1-3), 313-320.

[23] Fugetsu, B., Satoh, S., Iles, A., Tanaka, K., Nishi, N., and Watari, F., 2004, Encapsulation of multi-walled carbon nanotubes (MWCNTs) in $\mathrm{Ba}^{2+}$-alginate to form coated micro-beads and their application to the pre-concentration/elimination of dibenzo-pdioxin, dibenzofuran, and biphenyl from contaminated water, Analyst, 129 (7), 565-566.

[24] Luo, Y.B., Zheng, H.B., Wang, J.X., Gao, Q., Yu, Q.W., and Feng, Y.Q., 2011, An anionic exchange stir rod sorptive extraction based on monolithic material for the extraction of non-steroidal antiinflammatory drugs in environmental aqueous samples, Talanta, 86, 103-08.

[25] Manzo, V., Honda, L., Navarro, O., Ascar, L., and Richter, P., 2014, Microextraction of non-steroidal anti-inflammatory drugs from waste water samples by rotating-disk sorptive extraction, Talanta, 128, 486-492.

[26] Liu, L., Wen, Y.B., Liu, K.N., Sun, L., Wu, M., Han, G.F., Lu, Y.X., Wang, Q.M., and Yin, Z., 2013, Optimization of on-line solid phase extraction and HPLC conditions using response surface methodology for determination of WM- 5 in mouse plasma and its application to pharmacokinetic study, J. Chromatogr. B, 923-924, 8-15.

[27] Ahmad, M.A., and Alrozi, R., 2010, Optimization of preparation conditions for mangosteen peel-based activated carbons for the removal of Remazol Brilliant Blue $\mathrm{R}$ using response surface methodology, Chem. Eng. J., 165 (3), 883-890.

[28] Joglekar, A.M., and May, A.T., 1987, Product excellence through design of experiments, Cereal Food World, 32, 857-868.

[29] Funari, C.S., Carneiro, R.L., Cavalheiro, A.J., and Hilder, E.F., 2014, A trade off between separation, detection and sustainability in liquid chromatographic fingerprinting, J. Chromatogr. A, 1354, 34-42. 
[30] LoBrutto, R., Jones, A., Kazakevich, Y.V., and McNair, H.M., 2001, Effect of the eluent $\mathrm{pH}$ and acidic modifiers in high-performance liquid chromatography retention of basic analytes, $J$. Chromatogr. A, 913 (1-2), 173-187.

[31] Fugetsu, B., Satoh, S., Shiba, T., Mizutani, T., Lin, Y.B., Terui, N., Nodasaka, Y., Sasa, K., Shimizu, K., Akasaka, T., Shindoh, M., Shibata, K., Yokoyama, A., More, M., Tanaka, K., Sato, Y., Tohji, K., Tanaka, S., Nishi, N., and Watari, F., 2004, Caged multiwalled carbon nanotubes as the adsorbents for affinitybased elimination of ionic dyes, Environ. Sci. Technol., 38 (24), 6890-6896.
[32] Kot-Wasik, A., Dębska, J., Wasik, A., and Namieśnik, J., 2006, Determination of nonsteroidal anti-inflammatory drugs in natural waters using off-line and on-line SPE followed by LC coupled with DAD-MS, Chromatographia, 64 (1-2), 13-21.

[33] Paíga, P., Lolić, A., Hellebuyck, F., Santos, L.H.M.L.M., Correia, M., and Delerue-Matos, C., 2015, Development of a SPE-UHPLC-MS/MS methodology for the determination of nonsteroidal anti-inflammatory and analgesic pharmaceuticals in seawater, J. Pharm. Biomed. Anal., 106, 61-70. 